\title{
Surface states and origin of the Fermi level pinning on nonpolar $\mathrm{GaN}(1 \overline{1} 00)$ surfaces
}

L. Ivanova, S. Borisova, H. Eisele, M. Dähne, A. Laubsch, and Ph. Ebert

Citation: Appl. Phys. Lett. 93, 192110 (2008);

View online: https://doi.org/10.1063/1.3026743

View Table of Contents: http://aip.scitation.org/toc/apl/93/19

Published by the American Institute of Physics

\section{Articles you may be interested in}

Microscopic origins of surface states on nitride surfaces

Journal of Applied Physics 101, 081704 (2007); 10.1063/1.2722731

Two-dimensional electron gases induced by spontaneous and piezoelectric polarization charges in $\mathrm{N}$ - and Ga-face AIGaN/GaN heterostructures

Journal of Applied Physics 85, 3222 (1999); 10.1063/1.369664

The effect of polarity and surface states on the Fermi level at III-nitride surfaces

Journal of Applied Physics 116, 123701 (2014); 10.1063/1.4896377

Electronic surface and dielectric interface states on $\mathrm{GaN}$ and $\mathrm{AlGaN}$

Journal of Vacuum Science \& Technology A: Vacuum, Surfaces, and Films 31, 050807 (2013);

10.1116/1.4807904

Hidden surface states at non-polar GaN (10) 0$)$ facets: Intrinsic pinning of nanowires

Applied Physics Letters 103, 152101 (2013); 10.1063/1.4823723

Luminescence properties of defects in GaN

Journal of Applied Physics 97, 061301 (2005); 10.1063/1.1868059

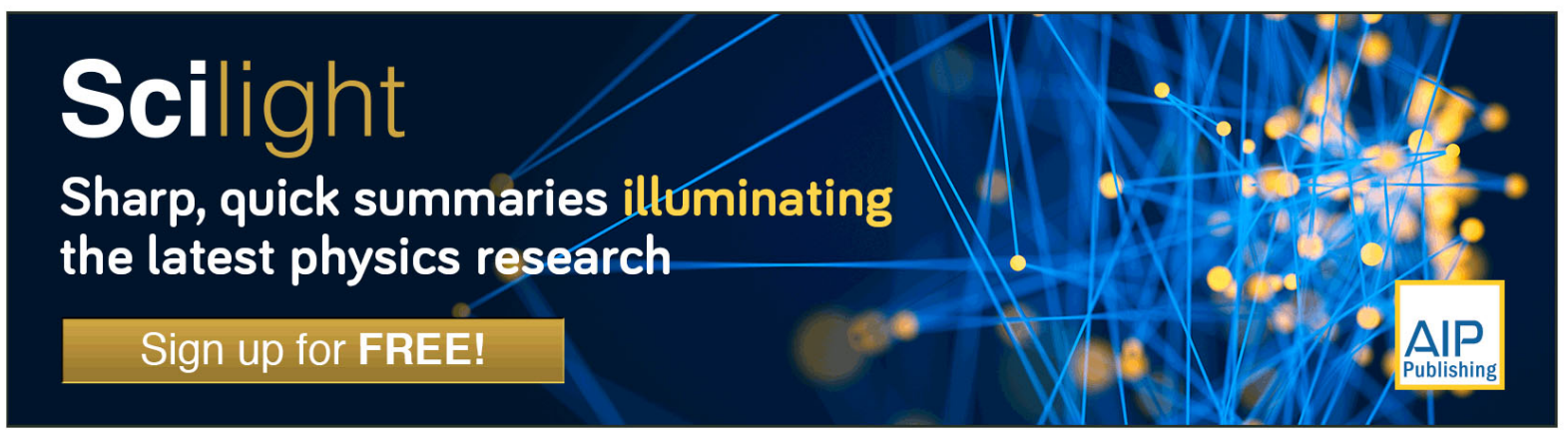




\title{
Surface states and origin of the Fermi level pinning on nonpolar GaN(1100) surfaces
}

\author{
L. Ivanova, ${ }^{1}$ S. Borisova, ${ }^{2}$ H. Eisele, ${ }^{1}$ M. Dähne, ${ }^{1}$ A. Laubsch, ${ }^{3}$ and Ph. Ebert ${ }^{2, a)}$ \\ ${ }_{1}^{1}$ Institut für Festkörperphysik, Technische Universität Berlin, Hardenbergstr. 36, 10623 Berlin, Germany \\ ${ }^{2}$ Institut für Festkörperforschung, Forschungszentrum Jülich GmbH, 52425 Jülich, Germany \\ ${ }^{3}$ OSRAM Opto-Semiconductors GmbH, Leibnizstrasse 4, 93055 Regensburg, Germany
}

(Received 2 October 2008; accepted 23 October 2008; published online 12 November 2008)

$\mathrm{GaN}(1 \overline{1} 00)$ cleavage surfaces were investigated by cross-sectional scanning tunneling microscopy and spectroscopy. It is found that both the $\mathrm{N}$ and Ga derived intrinsic dangling bond surface states are outside of the fundamental band gap. Their band edges are both located at the $\bar{\Gamma}$ point of the surface Brillouin zone. The observed Fermi level pinning at $1.0 \mathrm{eV}$ below the conduction band edge is attributed to the high step and defect density at the surface but not to intrinsic surface states.

(C) 2008 American Institute of Physics. [DOI: 10.1063/1.3026743]

Group-III nitrides raised considerable attraction because of their ideal properties for green, blue, and ultraviolet laser and light emitting devices. Therefore, intensive efforts have been invested to improve the quality of the epitaxial growth. One particular challenge is the impurity, dopant, and defect incorporation during growth, which often depends on the position of the Fermi level at the growth surface. ${ }^{1,2}$ Thus, it is of utmost importance to unravel the physical mechanisms governing the Fermi level position on group-III nitride surfaces. For the most commonly used polar $\mathrm{GaN}(0001)$ growth surface, surface states were identified as origin of the Fermi level pinning. ${ }^{3,4}$ In contrast, for the nonpolar GaN surfaces only little is known about the exact positions of the surface states and thus their possible influence on the Fermi energy. This is due to the lack of experimental data and disagreements between the existing theoretical calculations. Specifically, the energy positions of the surface states are inconclusive, as some calculations report that the empty dangling bond surface state lies within the fundamental bulk band gap, ${ }^{4-7}$ while others found no surface states in the band gap. ${ }^{8}$ It is, however, critical to know the exact energy position of surface states because this allows to identify which physical mechanism governs the Fermi energy at the surface, i.e., intrinsic surface state pinning, surface defect pinning, or bulk doping for unpinned surfaces. This lack of understanding is particularly embarrassing in the light that the growth along nonpolar GaN directions is very appealing due to the absence of electric fields caused by piezoelectricity and spontaneous polarization. ${ }^{9}$

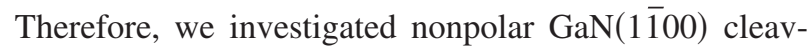
age surfaces by scanning tunneling microscopy (STM) and spectroscopy. We identify that no filled N or empty Ga derived dangling bond surface states are present within the fundamental band gap. The only spectroscopic feature within the band gap arises from electrons accumulated in defect states, pinning the Fermi energy at about $1 \mathrm{eV}$ below the conduction band minimum.

For our experiments we cleaved samples cut from $n$-type free-standing $\mathrm{GaN}(0001)$ single crystal substrates with a resistivity of about $(0.8-1) \times 10^{-2} \Omega \mathrm{cm}$ in ultrahigh

\footnotetext{
${ }^{\text {a) }}$ Author to whom correspondence should be addressed. Electronic mail: p.ebert@fz-juelich.de.
}

vacuum $\left(<10^{-8} \mathrm{~Pa}\right)$ along $(1 \overline{1} 00)$ planes. We used $\mathrm{Pt}_{0.8} \mathrm{Ir}_{0.2}$ tips.

Figure 1(a) illustrates the typical morphology of the $\mathrm{GaN}(1 \overline{1} 00)$ cleavage surface, which consists of atomically flat terraces separated by monoatomic steps. Low energy electron diffraction patterns [Fig. 1(b)] confirm a $1 \times 1$ surface unit cell. High resolution STM images, such as the one displayed in Fig. 1(c), show the atomic rows of the empty Ga dangling bonds along the [11 $\overline{2} 0]$ direction of the $1 \times 1$ ordered surface. This observed surface structure is in agreement with theoretical expectations ${ }^{8}$ and STM data of other cleaved wurtzite structure compound semiconductors. ${ }^{10}$

On terraces, such as those shown in Fig. 1(a), we measured the current-voltage spectra at different tip-sample separations (Fig. 2). At positive sample voltages all curves exhibit an onset of the current close to $+1 \mathrm{~V}$, while at negative voltages the onset strongly increases with increasing tip-

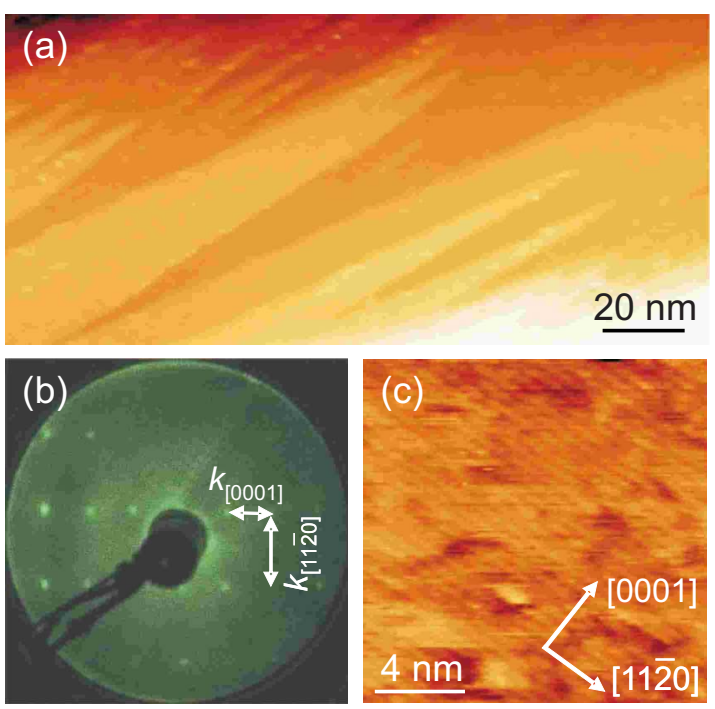

FIG. 1. (Color online) (a) Constant-current empty state STM image of a cleaved $\mathrm{GaN}(1 \overline{1} 00)$ surface measured at $+4.9 \mathrm{~V}$ sample voltage and $80 \mathrm{pA}$ tunnel current. The terraces are separated by monoatomic high steps. The orientation of the surface area is the same as in (c). (b) Low-energy electron diffraction pattern measured at $130 \mathrm{eV}$ consistent with a $1 \times 1$ surface structure. (c) STM image showing the atomic rows of empty Ga dangling bonds along the $[11 \overline{2} 0]$ direction (measured at $+5 \mathrm{~V}$ and $90 \mathrm{pA}$ ). 


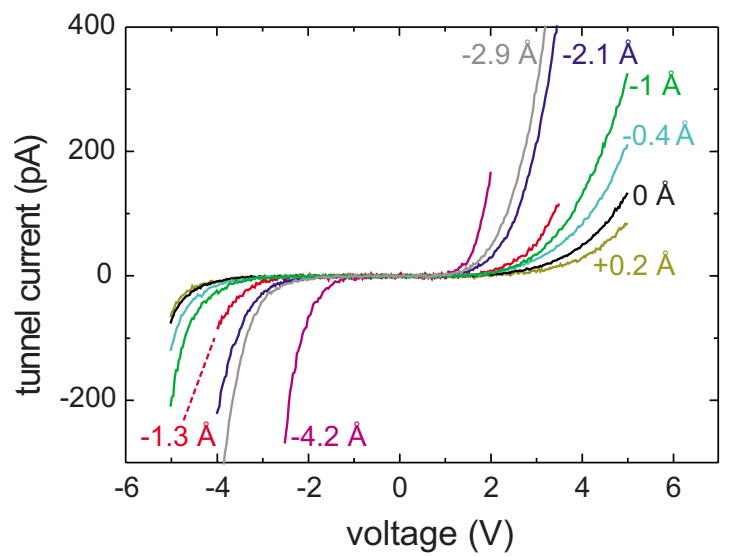

FIG. 2. (Color online) Current-voltage spectra measured on flat terraces of the cleaved $\mathrm{GaN}(1 \overline{1} 00)$ surface. The different curves correspond to different tip-sample separations $z=z_{0}+\Delta z$ ( $\Delta z$ is given at each curve and $z_{0}$ is defined by a set voltage and current of $+4.9 \mathrm{~V}$ and $80 \mathrm{pA}$, respectively).

sample separation. As a result the apparent band gap changes with the tip-sample separation and can be smaller than in bulk GaN. This raises the question where the band edges and the Fermi energy are positioned at the surface.

In order to identify the origins of the tunnel current and thus the positions of the band edges relative to the Fermi energy $E_{F}$, we turn to the logarithmic display of the current $I$ and the normalized differential conductivity $(d I / d V) /(\overline{I / V})$ as a function of the sample voltage (Fig. 3). The logarithmically displayed current curve in Fig. 3(a) exhibits (i) a clear onset at $+1.0 \mathrm{~V}$ of the tunnel current into the empty conduction band states of the surface $\left(I_{C}\right)$ and (ii) two different current contributions at negative voltages, i.e., $I_{\text {acc }}$ at voltages between 0 and $-2.5 \mathrm{~V}$ and $I_{V}+I_{\text {acc }}$ at voltages $V<-2.5 \mathrm{~V}$. The current contribution $I_{\text {acc }}$ is located at energies within the band gap of GaN. The different observed current contributions can be explained as follows:

First, at positive voltages the tunnel current $I_{C}$ flows if the Fermi level of the tip is energetically above the conduction band edge of the surface. Thus, the onset voltage at $+1.0 \mathrm{~V}$ corresponds to the surface $\left(E_{C}\right)$. This position of the conduction band edge indicates a Fermi level pinning of $1.0 \mathrm{eV}$ below $E_{C}$.

Second, tunnel currents $\left(I_{\text {acc }}\right)$ at voltages corresponding to energies within the fundamental band gap of a semiconductor were already observed previously on $\mathrm{GaAs}(110)$ surfaces. ${ }^{11-13}$ The observation of such tunnel currents requires filled semiconductor states to face empty tip states. This can only occur if (a) electrons accumulate at the GaN surface or (b) intrinsic surface states exist in the band gap. As discussed below, filled intrinsic surface states do not exist in the band gap and thus case (b) can be ruled out. One possibility of case (a) is if free charge carriers can accumulate in the conduction band near the GaN surface due to a tip-induced band bending. This would lead to filled conduction band states from which electrons can tunnel into the tip even at voltages corresponding to energies within the band gap. ${ }^{11-13}$ However, in our case, $E_{F}$ is pinned at $1 \mathrm{eV}$ below $E_{C}$ and thus the tip-induced band bending would have to be larger than $1 \mathrm{eV}$ in order to form an accumulation zone in the conduction band. Such large band bendings do, however, not occur at negative voltages $>-5 \mathrm{~V}$. Even on unpinned GaN surfaces the calculated band bending ${ }^{11,13}$ would be $<0.75 \mathrm{eV}$. Thus, $I_{\text {acc }}$ cannot arise from a tip-induced accu-

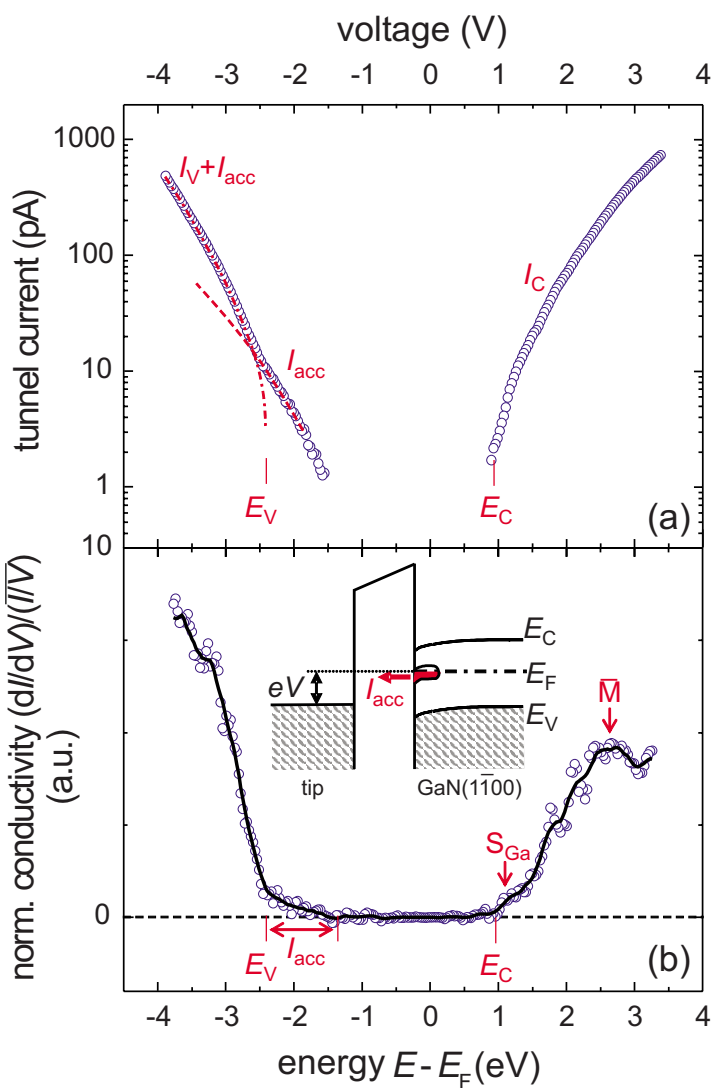

FIG. 3. (Color online) (a) Logarithmic display of the tunnel current as a function of voltage at a tip-sample separation of $z=z_{0}-3.3 \AA$. The conduction band $\left(I_{C}\right)$, accumulation $\left(I_{\text {acc }}\right)$, and valence band $\left(I_{V}\right)$ currents are indicated. The positions of the valence and conduction band edges $\left(E_{V}\right.$ and $\left.E_{C}\right)$ are marked. $E_{V}$ has been extracted from the onset of the $I_{V}$ current contribution (see dashed-dotted lines guiding the eyes). (b) Normalized differential conductivity $(d I / d V) /(\overline{I / V})$. The data points obtained by numerical differentiation of the current-voltage spectrum are shown as open circles. The solid line represents a smoothed curve. $S_{\mathrm{Ga}}$ marks the energetic minimum and $\overline{\mathrm{M}}$ marks the maximum DOS of the empty Ga dangling bond surface state. The band onsets $E_{V}$ and $E_{C}$ and the effect of the accumulation current $I_{\text {acc }}$ are clearly visible. Inset: schematic of the origin of the accumulation current. Note that only the effect of the tip-induced band bending is shown.

mulation zone in the conduction band. A further possibility of case (a) is the tip-induced accumulation of electrons in the states pinning the Fermi level (whose origin is discussed below). These states lie directly at the Fermi energy and even a very small tip-induced band bending can induce a carrier accumulation in these states, as schematically shown in the inset of Fig. 3(b). Therefore, we attribute the accumulation current $I_{\text {acc }}$ to this effect. Note that an analogous feature in the empty states at positive voltages is not observed because electrons tunneling into it simply fill the defect state and cannot be removed from there due to the lack of free holes (minority carriers) in $n$-type GaN. Thus, no current can be maintained into the surface bound defect state at positive voltages.

Third, if the magnitude of negative voltage is increased above the corresponding energy of the valence band edge $E_{V}$, also filled valence band states face empty tip states and additional electrons can tunnel yielding the $I_{V}$ current contribution. This effect leads to a second onset of the tunnel current close to $-2.5 \mathrm{~V}$ in Fig. 3(a), which thus corresponds to the valence band edge of the GaN surface $\left(E_{V}\right)$.

The energetic positions of $E_{V}$ and $E_{C}$ can be seen even better in the normalized differential conductivity 


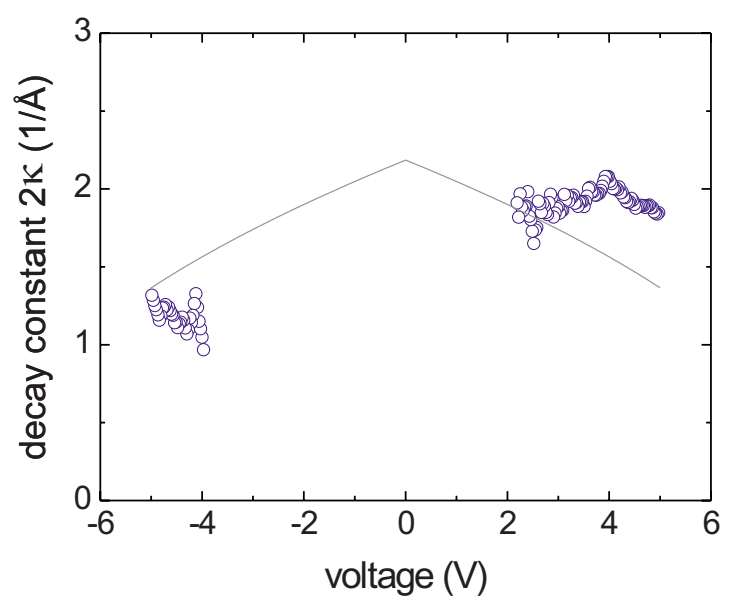

FIG. 4. (Color online) Decay constant $2 \kappa$ as a function of the voltage (open circles). The solid line shows the calculated trend for tunneling with zero parallel wave vector. These data reflect the dispersion of the surface states.

$(d I / d V) /(\overline{I / V})$ [Fig. 3(b)], ${ }^{14}$ which corresponds to the density of states (DOS). ${ }^{15}$ At $E_{F}-2.4 \mathrm{eV}$ and at $E_{F}+1.0 \mathrm{eV}$ clear onsets of the valence and conduction band DOS are visible, respectively. Thus, the band gap at the surface is $(3.4 \pm 0.2) \mathrm{eV}$ wide, matching well the bulk band gap of $\mathrm{GaN}$, supporting our identification of the band edges, and indicating the negligible effect of the tip-induced band bending on the derived energy positions.

The minimum of the empty Ga dangling bond surface state $\left(S_{\mathrm{Ga}}\right)$ can be attributed to the shoulder $0.1-0.2 \mathrm{eV}$ above $E_{C}{ }^{8}$ The large peak at $E_{F}+2.5 \mathrm{eV}[\overline{\mathrm{M}}$ in Fig. 3(b) $]$ can also be related to the flat part of the empty dangling bond's band dispersion around the $\overline{\mathrm{M}}$ point of the surface Brillouin zone, ${ }^{4,8}$ where the DOS is largest.

This can be further supported by an estimation of the momentum of the tunneling electrons through an analysis of the exponential decay of the tunnel current $I \sim e^{-2 \kappa z}$ with increasing tip-sample separation $z .{ }^{16}$ Figure 4 shows the measured decay constant $2 \kappa$ as a function of the voltage obtained for the largest tip-sample separations, where the effect of tip-sample interactions is negligible. The decay constant $2 \kappa$ can be approximated for one-dimensional systems and not too large voltages $V$ by ${ }^{13,15}$

$$
2 \kappa=2 \sqrt{\frac{2 m_{e}}{\hbar^{2}}\left(B-\frac{|e V|}{2}\right)+\left|k_{\|}\right|^{2}},
$$

with $m_{e}$ as the electron mass and $k_{\|}$as the parallel wave vector of the tunneling electrons. In the absence of a reliable three dimensional barrier model, we use this approximation to illustrate the expected trend as a function of the voltage, which is shown as solid line in Fig. 4 for $k_{\|}=0$ and an estimated effective tunneling barrier $B=4.3 \mathrm{eV}$. At negative voltages and at small positive voltages (around 2 to $3 \mathrm{~V}$ ) the measured values lie close to the calculated curve, indicating tunneling of electrons with $k_{\|}=0$. Thus, the $\mathrm{N}$ and $\mathrm{Ga}$ derived dangling bond surface states form a direct band gap at the $\bar{\Gamma}$ point. At larger positive voltages the decay constant is larger than expected for tunneling with $k_{\|}=0$. This indicates tunneling from the edge of the Brillouin zone, in agreement with the predicted dispersion of the empty surface state ${ }^{4-8}$ and the above interpretation of the peaks in the conduction band.

Finally, the accumulation current $I_{\text {acc }}$ and its $(d I / d V) /(\overline{I / V})$ signal are much smaller than those arising from the conduction and valence band states (Fig. 3). In addition, the current $I_{\mathrm{acc}}$ is more than four orders of magnitude smaller than the expected current from a tip-induced accumulation zone in the conduction band at the GaN surface, as calculated according to Ref. 11 for a direct band gap at the $\bar{\Gamma}$ point. This indicates that the filled DOS leading to $I_{\mathrm{acc}}$ is several orders of magnitude smaller than that of an accumulation zone in the conduction band. This is in good agreement with an origin of $I_{\text {acc }}$ in pinning states, whose concentration is typically in the range from $10^{11}$ to $10^{13} \mathrm{~cm}^{-2}$, i.e., much less than the DOS of the conduction band or of a dangling bond surface state. From these experimental observations we conclude that no intrinsic $\mathrm{N}$ and $\mathrm{Ga}$ derived dangling bond surface states are present in the fundamental band gap of $\mathrm{GaN}(1 \overline{1} 00)$ surfaces and the observed Fermi level pinning cannot arise from the dangling bond surface states. Instead it can be attributed to the large step densities of $10^{5}-10^{6} \mathrm{~cm}^{-1}$ and to point defects.

In conclusion, we identified the energy positions and types of surface states as well as the origin of the Fermi level pinning on $\mathrm{GaN}(1 \overline{1} 00)$ cleavage surfaces using crosssectional STM. We found that both the $\mathrm{N}$ and Ga derived dangling bond surface states are outside of the fundamental band gap. Their band edges are both situated at the $\bar{\Gamma}$ point of the Brillouin zone. The observed Fermi level pinning at $1 \mathrm{eV}$ below the conduction band edge is attributed to surface defects, but not to intrinsic surface states.

The authors thank the Deutsche Forschungsgemeinschaft for financial support as well as K. H. Graf and S. Landrock for assistance.

${ }^{1}$ D. G. Deppe, Appl. Phys. Lett. 56, 370 (1990).

${ }^{2}$ M. D. Pashley and K. W. Haberern, Phys. Rev. Lett. 67, 2697 (1991).

${ }^{3}$ A. R. Smith, R. M. Feenstra, D. W. Greve, M. S. Shin, M. Skowronski, J. Neugebauer, and J. E. Northrup, J. Vac. Sci. Technol. B 16, 2242 (1998).

${ }^{4}$ C. G. Van de Walle and D. Segev, J. Appl. Phys. 101, 081704 (2007).

${ }^{5}$ C. Noguez, Phys. Rev. B 62, 2681 (2000).

${ }^{6}$ A. Filippetti, V. Fiorentini, G. Cappellini, and A. Bosin, Phys. Rev. B 59, 8026 (1999).

${ }^{7}$ J. Wichert, R. Weber, L. Kipp, M. Skibowski, T. Strasser, F. Starrost, C. Solterbeck, W. Schattke, T. Suski, I. Grzegory, and S. Porowski, Phys. Status Solidi B 215, 751 (1999).

${ }^{8}$ J. E. Northrup and J. Neugebauer, Phys. Rev. B 53, R10477 (1996).

${ }^{9}$ P. Waltereit, O. Brandt, A. Trampert, H. T. Grahn, J. Menninger, M. Ramsteiner, M. Reiche, and K. H. Ploog, Nature (London) 406, 865 (2000).

${ }^{10}$ B. Siemens, C. Domke, Ph. Ebert, and K. Urban, Phys. Rev. B 56, 12321 (1997).

${ }^{11}$ N. D. Jäger, E. R. Weber, K. Urban, and Ph. Ebert, Phys. Rev. B 67, 165327 (2003).

${ }^{12}$ R. M. Feenstra, J. A. Stroscio, J. Tersoff, and A. P. Fein, Phys. Rev. Lett. 58, 1192 (1987).

${ }^{13}$ R. M. Feenstra and J. A. Stroscio, J. Vac. Sci. Technol. B 5, 923 (1987).

${ }^{14}$ M. Prietsch, A. Samsavar, and R. Ludeke, Phys. Rev. B 43, 11850 (1991).

${ }^{15}$ J. Tersoff and D. R. Hamann, Phys. Rev. Lett. 50, 1998 (1983); Phys. Rev. B 31, 805 (1985).

${ }^{16}$ J. A. Stroscio, R. M. Feenstra, and A. P. Fein, Phys. Rev. Lett. 57, 2579 (1986). 